\title{
Fitspiration to Inspire or to Mire? A Review of the Literature and Educational Implications
}

Joanne M. Crossman

\begin{abstract}
Fitspiration has been positioned as an online interchange featuring images and text designed to inspire fitness and healthy eating. Given its ubiquity as an emerging social media trend, and its unintended impact on body image disturbance mediated by social comparison, Fitspiration has been understudied. This review draws on theoretical perspectives associated with traditional and new media impact on young women's body image concerns, and overviews strategies to engage students in adopting a healthier body image. The manuscript also provides educational implications and directions for practice, particularly guided by media literacy models that may serve to intervene with body comparisons, inspire deeper learning and a renewed vision of fitness, health, and well-being.
\end{abstract}

\section{Background}

Body image research extended to newer media has recently included a preliminary exploration of fitness imagery and motivational phrases known as Fitspiration (an amalgamation of fit and inspiration). Fitspiration content is widely depicted on the Internet, and on social media platforms including Facebook, Instagram, Twitter, Tumblr, Pinterest, and Snapchat. The professed antidote for Thinspiration (photos and captions that glorify eating disorders), Fitspiration purportedly promotes health, well-being, and exercise, yet has been associated with female body image concerns; a relationship mediated by social comparison (Tiggemann \& Zaccardo, 2015; 2016; Vogel, 2015; Adams, 2014; Kite \& Kite, 2012; Abena University, 2013). Considering its prevalence and unintended negative influence upon our students' multimedia culture, Fitspiration has received limited review.

In fact, "little is known about women's responses to models presented in an active form, with a focus on athleticism and performance" (Mulgrew \& Hennes, 2014, p. 1). Therefore, with particular emphasis on Fitspiration content, this paper reviews literature, providing insights into the subtle effects that traditional media and social media exert on female body-perception, and overviews strategies to engage adolescent and young women in adopting a healthier body image. The manuscript also provides educational implications and directions for practice, particularly guided by media literacy models that may serve to intervene with body comparisons, inspire deeper learning and a renewed vision of fitness, health, and well-being. 


\section{Theoretical Perspectives: Media Influence on Body Concerns}

Extensive literature documents associations between media and female body dissatisfaction (Tiggemann \& Zaccardo, 2015; Perloff, 2014; Holland \& Tiggemann, 2016). Historically, female body image disturbances have been largely attributed to viewing thin, attractive models depicted in mass media, including magazines, TV ads, TV entertainment programs, movies, and music videos. Models have traditionally been portrayed in passive form with a focus on aesthetic qualities of the body. Much of the research was mediated by Objectification Theory (Fredrickson \& Roberts, 1997; McKinley \& Hyde, 1996), which purported that Western society sexually objectifies the female body for the pleasure and evaluation of others. Exposure to objectified images has long been associated with self-objectification and body dissatisfaction (Fredrickson \& Roberts, 1997; Harper, Sperry, \& Thompson, 2008). In turn, selfobjectification has been found to affect mental health, including a heightened risk for disordered eating, negative body esteem, and negative psychological well-being.

Numerous studies were also mediated by Bandura's (2009) Social Cognitive Theory, the Mass Communication-focused Cultivation Model (Morgan, Shanahan, \& Signorielli, 2009), and the Sociocultural Perspective on body image (Thompson, Heinberg, Altabe, \& Tantleff-Dunn, 1999; Tiggemann, 2011). These studies suggested media messages can impart unrealistic images of female beauty. Internalizing such distorted images has been associated with body dissatisfaction, a key predictor of disordered eating (Smolak \& Thompson, 2009; Ghaznavi \& Taylor, 2015).

Because there is a natural tendency for young people to compare themselves to peers and others, it is appropriate to consider Festinger's Social Comparison Theory as the central explanatory framework in which to place Fitspiration imagery and phrases purported to promote fitness. Social Comparison Theory maintained that people have the tendency to self-evaluate, self-enhance, and self-improve by comparing themselves to others. Whether the effects of such comparisons are positive/motivational or negative/demotivational may depend on whether one assimilates to, or contrasts away from the model of comparison. The theory further purported that people are primarily motivated to know themselves, and have a basic need to maintain a stable and accurate self-view through feedback about their performance and characteristics.

Festinger (1954) postulated that people self-evaluate by making lateral comparisons to others with standards similar to their own on particular critical dimensions. Next, downward comparisons serve as self-enhancement, create, and maintain a positive self-image. These comparisons are made with others we outperform. A third need fulfilled by social comparison is self-improvement. Here, people seek upward comparisons of standards depicted by others we perceive to be superior.

For example, assuming that comparing oneself to an idealized, thin model constitutes an upward comparison and to a heavy model, a downward comparison, we can consider assimilation effects and contrast effects. Mathras, Loveland, and Mandel (2013) suggested that comparing weight and shape with a moderately thin model may garner assimilation effects towards a possibly attainable ideal. However, 
contrast effects away from dramatically thin models have largely been associated with negative selfesteem and low body satisfaction.

Attaining an accurate self-view towards improved fitness and overall health and well-being may be both warranted and useful. Yet, comparisons to a false, unattainable fitness ideal created by "manicuring the online self...to adhere to the mainstream aesthetics," could lead to a sense of inadequacy when failing to live up to idealized online personas (Manago, Ward, Lemm, Reed, \& Seabrook, 2014, p. 10). Such comparisons can influence thin-ideal ideation, body dissatisfaction, and eating pathology.

\section{Social Media and Body Concerns: Thin-Ideal Internalization, Body Dissatisfaction, and Eating Pathology}

During its 2015 survey period, Statista found in North America, 16-24 year olds spent approximately 200 minutes daily on mobile devices. Among high school graduates, when using mobile devices, the most popular social networks and apps included text messaging, followed by Instagram, then Facebook and Snapchat (Statista, 2016a). Statista's (2016b) worldwide data ranked by number of active users showed approximately 2 billion Internet users utilize social networks in highly diverse platforms including Facebook, and microblog rapid communications such as Tumblr or Twitter. Some social networks focus on community; others highlight and display user-generated content.

The most popular network worldwide as of April 2016, was Facebook with 1.59 billion monthly active users. Eighth-ranked photo-sharing app Instagram had over 400 million monthly active accounts. Blogging service Tumblr had more than 555 million active bloggers. Due to a constant presence in their users' lives, social networks have a decidedly strong social impact. The blurring between offline and virtual life as well as the concept of digital identity and online social interactions are some aspects that have emerged in recent discussions (Statista, 2016b).

It is therefore increasingly important to study potential relationships between social media use and body image concerns. These relationships continue to be aligned with appearance comparisons (Fardouly \& Vartanian, 2015; Fardouly, Diedrichs, Vartanian, \& Halliwell, 2015a; Fardouly et al., 2015b; Kim \& Chock, 2015; Lewallen \& Behm-Morawitz, 2016). Most studies of traditional and social media impact on body image and disordered eating have been conducted using female samples. However, in their review of 20 studies investigating the influence of social media on body image and disordered eating, Holland and Tiggemann (2016) found disturbances reported among both males and females. The majority of these studies focused on undergraduate or high school students, whom they contended are prone to such ideation. Measures included overall usage time, frequency of use, and number Facebook "friends." Disturbingly, Holland and Tiggemann (2016) also reported that longitudinal studies of social media use indicated by these measures resulted in increased levels of body image concerns from four to 18 months later.

Perloff's 2014 research further suggested that women low in self-esteem and high in perfectionism, appearance-based self-worth, or thin-ideal internalization will turn to social media for reassurance and 
validation regarding physical and social attractiveness. Peer-based social media contexts, he contended, invite social comparison to attractive peers and the thinness ideal.

Indeed, imagery, text, and Thinspiration content have been shown to exacerbate thin-ideal internalization, body dissatisfaction, and eating pathology. A disquieting trend, Thinspiration (an amalgamation of thin and inspiration) is a confluence of images depicting emaciated women accompanied by quotes designed to inspire weight loss and promote an eating-disordered lifestyle (Borzekowski, Schenk, Wilson, \& Peebles 2010; Ghaznavi \& Taylor, 2015). Thinspiration promotes weight loss in a manner that glorifies dangerous behaviors consistent with eating disorders (Lewis \& Arbuthnott, 2012; National Eating Disorders Association, 2016).

For example, the Pro ANA Lifestyle Forever webpage (n. d.) readily available in November 2016 featured the ANA (Anorexia) Religion and Lifestyle Thin Commandments:

1. If you aren't thin, you aren't attractive

2. Being thin is more important than being healthy

3. You must buy clothes, cut your hair, take laxatives; anything to make yourself look thinner

4. Thou shall not eat without feeling guilty

5. Thou shall not eat fattening food without punishing afterwards

6. Thou shall count calories and restrict intake accordingly

7. What the scale says is the most important thing

8. Losing weight is good, gaining weight is bad

9. You can never be too thin

10. Being thin and not eating are signs of true willpower and success.

Perloff (2014) argued that thinness-inspiring websites, blogs, and images like Thinspiration specifically promote eating disorders, including anorexia and bulimia. Perloff's research found that Facebook, Instagram, and Pinterest, "allow for creating and exchange of messages on particular body image-related content, exemplified by Thinspiration blogs and pro-eating disorder material" (p. 368). Mabe, Forney, and Keel (2014) noted social media, "provides a constant, active space to engage in social comparison with peers who may simultaneously portray and reinforce the thin-ideal" (p. 520). They found women with greater eating pathology reported engaging in appearance-focused behaviors such as comparing their appearance with friends' pictures and untagging their own unflattering photos, perhaps so as to avoid the possibility of others' making downward comparisons with them.

Prieler and Choi (2014) argued it is necessary to differentiate social media use driven by entertainment or socializing, with use driven by specific body image needs and vulnerabilities. For example, a young woman using social media primarily for socializing may encounter pictures of thin women posted by her peers. That content may be more or less influential in eliciting body dissatisfaction depending on her vulnerability factors (level of self-esteem or the centrality of appearance to her self-worth). In contrast, individuals fixated on the thinness ideal may use social media to seek gratification specific to their body 
image concerns. By encountering pro-eating disorder sites, they may experience greater dissatisfaction, yet also a positive effect through social support from like-minded individuals (Csipke \& Horne, 2007).

Given their propensity to engage in social media's interactive format and content, young adults, particularly women, can be influenced by negative social comparisons, and peer normative processes, thereby significantly influencing body image concerns (Perloff, 2014).

For example, Lewallen and Behm-Morawitz (2016) described Pinterest as a feminized social media form: "a content curation website analogous to an online vision board" (p. 6) where users may collect and share images towards upward social comparison. They found the sum of fitness-focused pinboards followed by study participants predicted whether they were influenced to engage in extreme weight-loss tactics such as fad dieting.

Williams and Ricciardelli (2014) argued that social media exposes users to

unrealistic body images, modelling, pressure to conform, gender-typed socialization, objectification of the body, internalization of appearance ideals, increased negative affect that results from viewing unrealistic images of the body, social comparisons, interactions with peers and other normative influences, behaviors and body change strategies to improve oneself, and compensatory motivations such as disordered eating as a way to validate one's self-concepts. (p. 2)

However, Williams and Ricciardelli (2014) asserted the processes underlying the ways social media promotes body image concerns appear to be no different from other media forms. In fact, they contended it is not yet known if social media exacerbates the effects of these processes because the images are omnipresent, or if we have become desensitized and pay less attention to the messages.

Yet, various unique characteristics of social media may contribute to any effects it has on body image. For example, social media features the users themselves, in addition to models and celebrities. Also, users often upload the most attractive images of themselves (easily edited and enhanced), and remove those they perceive as unattractive (Zhao, Grasmuck, \& Martin, 2008; Fardouly \& Vartanian, 2016). Therefore, social media provides a digital platform for users to present the version of themselves they want their social network to see (Zhao et al., 2008). "Users become vigilant about how they will appear to others as they reflect, edit, and manicure self-portrayals for an audience of followers" (Manago et al., 2014 , p. 10). Consequently, such edited, enhanced images may convey idealized images of social peers (Kim \& Chock, 2015).

Social media may also reinforce the thin-ideal internalization through various posts, "likes," and comments related to appearance, also possibly affecting how users feel about their appearance (Carey, Donaghue, \& Broderick, 2014; Mabe et al., 2014). Relative to content on Twitter and Pinterest, Ghaznavi and Taylor (2015) suggested thin-ideal images provide examples for self-comparison and could perpetuate belief that these bodies are relatively more common than others, thereby associating disordered eating attitudes and body dissatisfaction. Moreover, social media is generally used to interact 
with one's peers, and peers have been shown to influence risk for body dissatisfaction and eating pathology (Zalta \& Keel, 2006; Keel, Forney, Brown, \& Heatherton, 2013).

Social media users actively search for contact, forge social ties, and seek information about others, (Tufekci, 2008). This is known as "social grooming" (Dunbar, 1996; Tufekci, 2008). Social grooming behaviors such as checking friends' profiles, commenting, and leaving messages may increase opportunities for viewing enhanced images, thereby leading to a greater likelihood of comparing one's appearance to others. This social grooming may correlate with body image concerns more strongly than simple exposure time (Kim \& Chock, 2015). The comparisons are often made with people who strategically portray themselves in the most positive fashion, such as those depicted in Fitspiration imagery.

\section{Fitspiration: Negative, Unintended Impact on Body Image Mediated by Social Comparison}

Unlike Thinspiration, Fitspiration (an amalgamation of fit and inspiration) was purportedly designed to inspire a strong and positive body image through exercise and healthy eating. Fitspo imagery is widely available on the Internet, and Tiggemann and Zaccardo (2015) found it most notably present on Instagram, which is often linked to other social media accounts, thereby increasing dissemination of this imagery. In fact, Instagram users may be subject to fitness-related messages they did not even opt to see when their peers post pictures, or if a friend "liked" an image on Facebook.

On Tumblr, users can find images by searching for tags like "Fitspo," on Pinterest, users can pin Fitspiration images and text, and on Facebook users can share pictures of self and peers. Fitspiration imagery may also target advertising. Stieben (2013) explained that targeted social media advertisements are garnered through users' profile information, location, and browsing history.

Food or exercise mentioned on social media may result in users being presented with related advertisements.

Adams (2014) described Fitspiration as an online community of bloggers and social media accounts that encourage weight loss, diet, and exercise by sharing success stories, active lifestyle and diet tips, photos, and rules. Tiggemann and Zaccardo (2015) contended, "Overall health and well-being are strongly endorsed through promotion of healthy eating, exercise, and self-care. The general philosophy is one emphasizing strength and empowerment, and strong is the new skinny" (p. 62).

Conversely, health blogger, Arnot (2016) argued Fitspo is actually making us less active. She cautioned that beginners who have never done much physical activity; those insecure about their body, embarrassed or unsure; those who believe they are uncoordinated or not sports-like; the ill, injured, or disabled; and the vast majority of people who cannot identify with Fitspo models find Fitspiration so overzealous, un-relatable, so all or nothing that they choose nothing. 
Additionally, simply searching "fitness" or "Fitspo" may lead users to Thinspiration content (Ghaznavi \& Taylor, 2015). Fitspiration images posted on Instagram also share common characteristics found to be negative for body image. In particular, the clear overrepresentation of the ideal body shape for women and men, the overall focus on appearance, and the presence of objectifying features have been linked to poorer body image and disordered eating (Tiggemann \& Zaccardo, 2016).

Moreover, the majority of women in the images were found to have a generally unattainable tone, relatively thin figure, albeit less thin and more muscular than models portrayed in fashion magazines. They cautioned this particular fitness-figure implies only thin and toned bodies can be fit, contending Fitspiration imagery attempts to inspire women toward health and fitness by focusing on appearancerelated body conceptualization (Tiggemann \& Zaccardo, 2015).

Body Conceptualization Theory suggests the body may be evaluated in two ways: BAO or BAP (Franzoi, 1995). Viewing the body as an object (BAO), the aesthetic value of its discrete parts is emphasized. Viewing the body as a process (BAP), the functional attributes (strength, flexibility, and endurance) are emphasized. Abbott and Barber (2010) found women place great value on aesthetic qualities of their bodies (BAO), correlating with lower self-esteem and increased depressed mood. However, greater value placed on the body's functional aspects was positively related to self-esteem. Similarly, Strelan, Mehaffey, and Tiggemann (2003) found exercise motivated by appearance, rather than health or enjoyment, was associated with negative body image.

Mulgrew and Hennes (2014) surmised that females who viewed images of thin and attractive models reported similar and negative outcomes regardless of focus on form or function. Detrimental effects (greater depression, anger, and feelings of fatness) were reported by women with a high desire to emulate the athletic physique after viewing images of models, compared to women who expressed less desire for an athletic body shape.

These findings suggest that images with a focus on BAP appear to elicit reflection on the object dimensions of a body. Fitspiration images often have objectifying features such as a focus on particular body parts (Tiggemann \& Zaccardo, 2015).

Arnot (2016) recommended unfollowing, hiding, and deleting Fitspo messaging that makes us feel anxious, insecure, alienated, or obsessive, and commenting to companies rebuking unrealistic marketing strategies. She advocated choosing movement that is enjoyable, feels good, improves function, or increases competence at something we enjoy. Arnot contended progress and appearance are disparate, and movement is only one element of good health, which takes many forms.

Recently, Mulgrew (2016) argued displaying images of overly fit females to promote exercise could be counterproductive, refuting the common assumption that Fitspiration photographs promote a healthier body image. Participants in her study who viewed fitness model imagery reported feeling worse about themselves than did those who viewed traditional models. Mulgrew concluded that fitness images should not be assumed less harmful to body image, cautioning that women should value their body's functionality beyond appearance. 
In sum, it appears fitness imagery has not evolved beyond the one same body type: slim, toned, and attractive. Tiggemann and Zaccardo (2015) contended, "Exposure to Fitspiration leads to negative body image, and this relationship is mediated by social comparison... Fitspiration avowedly designed to promote healthy eating and exercise can have (unintended) negative consequences on body image" (p. 66).

\section{Regulations, Disclaimers, and Referrals}

While regulating health and fitness-related social media content may be an option for dealing with potentially harmful effects, doing so is challenging (Carrotte, Vella, \& Lim, 2015). Some Thinspiration communication has been banned or censored, with varying degrees of success. Spiegel (2013) rated poorly the big social networks' enforcement of banned pro-eating disorder content. For example, Instagram (D rating) rendered \#Thinspiration, \#probulimia, and \#proanorexica tags unsearchable. Yet tags such as \#thinspire are easily searched there, many portraying images of skeletal bodies and cutting.

Various disclaimers and content advisories are offered by social networks such as Tumblr and Instagram, as are referrals to links including the National Eating Disorders Association (NEDA) website and various recovery resources. NEDA has worked with many major social media platforms to help develop community guidelines, and to identify Thinspo and Fitspo content promoting disordered eating or selfharm behavior. For example, NEDA (2016) recommended that media platforms highlight the dangers, but avoid sensationalizing the issue.

Help your readers to understand that pro-anorexia and pro-bulimia sites are very dangerous, both for those who have an eating disorder and those who do not, but may be vulnerable:

Research indicates providing numbers for lowest weights or goal weights, and showing graphic images glorifying extreme thinness can be triggering. Individuals struggling with an eating disorder might believe they need to reach the published "low weights" before qualifying for getting help. Individuals susceptible can also use this number as a goal weight and sink deeper into illness. Publishing calorie counts and detailing specific behaviors for weight loss are also dangerous... and could result in behaviors that put the individual at serious risk for health complications, further entrenching the eating disorder (NEDA, 2016).

However, specific guidelines for health and fitness-related social media content are not immediately apparent, although some platforms specifically admonish imagery and text relevant to disordered eating. In part, these general standards were available at the time of this review.

Facebook Community Standards (2016)

We don't allow the promotion of self-injury or suicide. We work with organizations around the world to provide assistance for people in distress. We prohibit content that promotes or encourages suicide or any other type of self-injury, including self-mutilation and eating disorders... 
Instagram Community Guidelines (2016)

The Instagram community... is often a place where people facing difficult issues such as eating disorders, cutting, or other kinds of self-injury come together to create awareness or find support. We try to do our part by providing education in the app and... in the Help Center...Encouraging or urging people to embrace self-injury is counter to this...support, and we'll remove it or disable accounts if it's reported to us. We may also remove content identifying victims or survivors of self-injury if the content targets them for attack or humor.

The Twitter Rules (2016)

You may encounter someone considering suicide or self-harm on Twitter. When we receive reports that a person is threatening suicide or self-harm, we may take a number of steps to assist them, such as...expressing our concern and the concern of other users on Twitter or providing resources such as contact information for our mental health partners.

Tumblr Community Guidelines (2015)

Don't post content that actively promotes or glorifies self-harm... or encourages others to: cut or injure themselves; embrace anorexia, bulimia, or other eating disorders; or commit suicide rather than seeking counseling or treatment...online communities can be extraordinarily helpful to people struggling with these difficult conditions. We aim for Tumblr to...facilitate awareness, support and recovery, and we will remove only those posts or blogs that cross...into active promotion or glorification of self-harm.

Pinterest Acceptable Use Policy (2016)

Pinterest is a place to get inspired...promoting dangerous behavior detracts from that. We don't allow anything that promotes self-harm, eating disorders or hard drug abuse. Sometimes people want to share their experiences... as a way of helping others who are struggling...We just don't want to encourage harmful behaviors.

Snapchat Community Guidelines (2016): "What not to Snap: Threats- Intimidating people is not okay. Harassment or bullying-...Do not make other people feel bad on purpose."

Carrotte et al. (2015) argued these general guidelines may not adequately identify harmful content, particularly because problematic health and fitness messages may be subtle, or tagged as "healthy." In essence, social media platforms subtly bypass their own recommendations by disseminating Fitspiration content that disregards National Eating Disorders Association guidelines. See NEDA, 2016.

Clearly, consuming and producing Fitspiration-type media has become a normative aspect of adolescent and young adult life. Disturbingly, however, even images and messages focused on flexibility and strength appear to elicit negative self-evaluation among female media consumers (Mulgrew \& Hennes, 2014). Moreover, as media producers, one's pictures and posts related to food and exercise (although possibly edited or enhanced) ostensively shape their personal brand (Vaterlaus, Patten, Rochec, \& Young, 2015). It is therefore critical to "help young people develop critical and abstract thinking skills to consider carefully how they portray themselves in new media" (Daniels, 2016, p. 10) and to understand the nature, techniques, and impacts of media messages. 


\section{Educational Implications}

The preceding review well illustrates the complexity of social media in body image concerns; a relationship mediated by social comparison. The proliferation of health and fitness-related social media suggests, "its messages are unavoidable... and easily normalized regardless of actual health benefits" (Carrotte et al., 2015, p. 8).

Unattainable, digitally edited standards of beauty reinforced by images circulated by one's peers makes it extremely challenging for young women to feel satisfied with their own physical appearance. However, because social media affords opportunity for users to generate content, educators and students can use this medium to extend social media literacy, counter social comparison, and create and advocate positive/inclusive images of health, each towards fulfilling Fitspiration's purported purposes (promoting exercise and healthy eating).

This reinforces the need and importance of media literacy education; specifically, education that fosters wellness over appearance, and inspires informed, responsible, social media consumers and creators. "The available evidence suggests body image and media literacy intervention programs should address the potential impact of social media on its users" (Fardouly \& Vartanian, 2016, p. 3). Holland and Tiggemann (2016) added, "...especially the pressures associated with the uploading and viewing of images" (p. 108).

To support uptake or maintenance of media literacy education, teachers must first understand it. Indeed, Cahil, Lester, Midford, Ramsden, and Venning (2014) found significant influences on educators' pedagogical choices included understanding the educational rationale for an approach. Jeong, Cho, and Hwang (2012) provided such rationale in their meta-analysis of media literacy interventions, finding those most impactful can enhance both media-relevant and behavior-relevant outcomes.

Tiggemann and Zaccardo (2015) noted, "Media literacy programs which have shown some success in combating negative body image need to be expanded to incorporate social networking media in general, and Fitspiration imagery in particular" (p. 66). In fact, both men and women might usefully be educated about potential body dissatisfaction and effects from exposure to Fitspiration images (Tiggemann \& Zaccardo, 2016). Education can help individuals recognize risks of social comparison in online and social media contexts (Lewallen \& Behm-Morawitz, 2016). In particular, women should be warned against considering females portrayed in Fitspiration imagery as aspirational targets for social comparison (Adams, 2014).

Regardless of the particular platform, various factors in social media environments (i.e., featuring users themselves, ease of editing/enhancing images) may influence social comparison or self-enhancing tactics, including extreme dieting or exercise. Exploring such factors through media education may enhance students' skills in effectively evaluating the credibility, quality, risks, and benefits of health information and Fitspiration imagery. 


\section{Media Literacy Resources}

A promising intervention, the Get Real: Digital Media Literacy Toolkit addresses potential influences (i.e., Fitspiration) on normalizing unrealistic body standards. Partnering with the NEDA, California State University students designed the toolkit, engaging university/high school students in testing their media literacy skills, discovering what their digital footprint reveals about their body image, and taking a stand against implausible, potentially harmful body portrayals. The toolkit also provides guidance for writing "social media shout-outs" to celebrities who oppose retouched photos of themselves. By using media literacy skills framed by media-relevant/behavior-relevant outcomes to advocate for change, the toolkit's resources can "shift the spotlight from limited/artificial body ideals to more diverse and authentic body shapes and sizes that reinforce healthy lifestyles" (NEDA \& California State University, n. d.).

Key Concepts of Media Literacy is another promising resource originating from MediaSmarts (2015), formally the Media Awareness Network. The Canadian not-for-profit organization designed this tool to encourage students to think critically and ethically, and reflect about their multimedia culture. With direct relevance to social comparison and body dissatisfaction, Key Concepts engages students as contributors to public debate about Fitspiration; deepens understanding of diversity, identity, and difference; explores connections between popular culture and attitudes, lifestyle choices and self-image; assists with critique of media representations so as to distinguish between reality and fantasy; and develops critical thinking, evaluation, and authentication skills. "Digital literacy enables individuals to understand how digital media content and applications can reflect, shape, enhance or manipulate our perceptions, beliefs and feelings" (p. 5).

MediaSmarts (2015) contended critical media consumers have a command of concepts necessary to examine media: Media are constructions; Audiences negotiate meaning through individual factors; Media have commercial implications-even in content not made for profit, distribution is generally facilitated by profit intentions; Media have social and political implications; and each medium has a unique aesthetic form.

Finally, Vogel's (2015) Positive Body Image Posting Guide encourages Fitspo contributors to reflect before posting, posing questions such as, "Am I posting this message or photo? ...for my own gratification or to inspire, help and connect with my network?" (p. 1). The (U.S.) National Action Plan to Improve Health Literacy (2010), Healthy Campus 2020, and numerous Body Positive Campaigns are also impactful for young adults.

As social media evolves, educators must address it both as a place for fluid expression and as an environment with potential to perpetuate stereotypes, dangerous body ideals, and social comparison; a place where health and well-being trends such as Fitspiration are less likely to inspire, and more likely to mire.

Future research could draw on sociology of health or physical education literature. Case study investigation of behavior-related outcomes affected through a featured media literacy program may broaden understanding of how they function to buffer social comparisons inherent to Fitspiration. 


\section{References}

Abbott, B. D., \& Barber, B. L. (2010). Embodied image: Gender differences in functional and aesthetic body image among Australian adolescents. Body Image, 7, 22-31. doi:10.1016/j.bodyim.2009.10.004

Abena University of Chicago. (2013). From Thinspo to: How social media could be affecting your body image. Retrieved from http://www.collegefashion.net/college-life/from-thinspo-to-Fitspiration-howsocial-media-could-be-affecting-your-body-image/

Adams, R. (2014). Why 'Fitspo' should come with a warning label. Retrieved from http://www.huffingtonpost.com/2014/07/17/Fitspo-Fitspiration_n_5574150.html

Arnot, J. (2016). Is 'Fitspo' making us less active? Healthy Balance Fitness (company). Retrieved from http://www.healthybalancefitness.com.au/2016/03/is-Fitspo-making-us-less-active/

Bandura, A. (2009). Social cognitive theory of mass communication. In J. Bryant \& M. B. Oliver (Eds.), Media effects: Advances in theory and research (pp. 94-124). New York: Routledge.

Borzekowski, D. L., Schenk S., Wilson, J. L., \& Peebles, R. (2010). E-Ana and e-Mia: A content analysis of pro-eating disorder web sites. American Journal of Public Health, 100, 1526-1534.

Cahil, H., Lester, L., Midford, R., Ramsden, R., \& Venning, L. (2014). Influences on teachers' use of participatory learning strategies in health education classes. Health Education Journal, 73, 702-713.

Carey, R. N., Donaghue, N., \& Broderick, P. (2014). Body image concern among Australian adolescent girls: The role of body comparisons with models and peers. Body Image, 11(1), 81-84. doi:10.1016/j.bodyim.2013.09.006.

Carrotte, E. R., Vella. A. M., \& Lim, M. (2015). Predictors of "liking" three types of health and fitnessrelated content on social media. Journal of Internet Medical Research, 17(8). Retrieved from http://www.ncbi.nlm.nih.gov/pmc/articles/PMC4642410/

Csipke, E., \& Horne, O. (2007). Pro-eating disorder websites: User's opinions. European Eating Disorders Review, 15, 196-206. doi:10.1002/erv.789

Daniels, E. (2016). The price of sexy: Viewer's perceptions of a sexualized versus nonsexualized Facebook photograph. Psychology of Popular Media Culture, 51(1), 2-14.

Dunbar, R. M. (1996). Grooming, gossip, and the evolution of language. Boston, MA: Harvard University Press.

Facebook Community Standards. (2016). Self-Injury: How we work to help prevent self-injury and suicide. Retrieved from https://www.facebook.com/communitystandards

Fardouly, J., Diedrichs, P. C., Vartanian, L. R., \& Halliwell, E. (2015a). Social comparisons on social media: The impact of Facebook on young women's body image concerns and mood. Body Image, 13, 38-45. Retrieved from http://www.sciencedirect.com/science/article/pii/S174014451400148X

Fardouly, J., Diedrichs, P. C., Vartanian, L. R., \& Halliwell, E. (2015b). The mediating role of appearance comparisons in the relationship between media usage and self-objectification in young women. Psychology of Women Quarterly. Retrieved from http://dx.doi.org/10.1177/0361684315581841 
Fardouly, J., \& Vartanian, L. R. (2015). Negative comparisons about one's appearance mediate the relationship between Facebook usage and body image concerns. Body Image, 12, 82-88. Retrieved from http://dx.doi.org/10.1016/j.bodyim.201410.004.

Fardouly, J., \& Vartanian, L. R. (2016). Social media and body image concerns: Current research and future directions. Current Opinion in Psychology, 9, 1-5. Retrieved from http://www.sciencedirect.com

Festinger, L. (1954). A theory of social comparison processes. Human Relations, 7, 117-140. Retrieved from http://www.uni-bielefeld.de/ikg/zick/Festinger\%20(1954).pdf

Franzoi, S. L. (1995). The body-as-object versus the body-as-process: Gender differences and gender consideration. Sex Roles, 33, 417-437. doi:10.1007/BF01954577.

Fredrickson, B. L., \& Roberts, T. A. (1997). Objectification theory: Toward understanding women's lived experiences and mental health risks. Psychology of Women Quarterly, 21, 173-206. doi:10.1111/j.1471-6402

Ghaznavi, J., \& Taylor, L. D. (2015). Bones, body parts, and sex appeal: An analysis of \#Thinspiration images on popular social media. Body Image, 14, 54-61.

Harper, K., Sperry, S., \& Thompson, J. K. (2008). Viewership of pro-eating disorder websites: Association with body image and eating disturbances. International Journal of Eating Disorders, 41, 92-95.

Holland, G., \& Tiggemann, M. (2016). A systematic review of the impact of the use of social networking sites on body image and disordered eating outcomes. Body Image, 17, 100-110.

Instagram Community Guidelines. (2016). Retrieved from

https://help.instagram.com/477434105621119/

Jeong, S. E., Cho, H., \& Hwang, Y. (2012). Media literacy interventions: A meta-analytic review. Journal of Communication, 62(3), 454-472. doi:10.1111/j.1460-2466.2012.01643.x

Keel, P. K., Forney, K. J., Brown, T. A., \& Heatherton, T. F. (2013). Influence of college peers on disordered eating in women and men at a 10-year follow up. Journal of Abnormal Psychology, 122(1), 105-110.

Kim, J. W., \& Chock, T. M. (2015). Body image 2.0: Associations between social grooming on Facebook and body image concerns. Computers in Human Behavior, 48, 331-339. Retrieved from http://dx.doi.org/10.1016j.chb.2015.01.009

Kite, L., \& Kite, L. (2012). Why Fitspiration isn't so inspirational. Beauty redefined. Retrieved from http://www.beautyredefined.net/why-Fitspiration-isnt-so-inspirational/

Lewallen, J., \& Behm-Morawitz, E. (2016). Pinterest or Thinterest? Social comparison and body image on social media. Social Media + Society, 2(1). doi:10.1177/2056305116640559

Lewis, S. P., \& Arbuthnott, A. E. (2012). Searching for Thinspiration: The nature of internet searches for pro-eating disorder websites. Cyberpsychology, Behavior, and Social Networking, 15, 200-204. Retrieved from http://dx.doi.org/10.1089/cyber.2011.0453

Mabe, A. G., Forney, K. J., \& Keel, P. K. (2014). Do you "Like" my photo? Facebook use maintains eating disorder risk. International Journal of Eating Disorders, 47(5), 516-523. 
Manago, A. M., Ward, L. M., Lemm, K. M., Reed, L., \& Seabrook, R. (2014). Facebook involvement, objectified body consciousness, body shame, and sexual assertiveness in college women and men. Sex Roles, 72, 1-14. doi:10.1007/s11199-014-0441-1

Mathras, D., Loveland, K. E., \& Mandel, N. (2013). Media image effects on the self. In A. A. Ruvino, \& R. W. Belk (Eds.), The Routledge companion to identity and consumption (pp. 385-395). New York: Routledge.

McKinley, N. M., \& Hyde, J. S. (1996). The objectified body consciousness scale: Development and validation. Psychology of Women Quarterly, 20, 181-215. doi:10.1111/j.1471-6402

MediaSmarts. (2015). Mapping digital literacy policy and practice in the Canadian education landscape. Retrieved from http://mediasmarts.ca/sites/mediasmarts/files/publication-report/full/mapping-digitalliteracy.pdf

Morgan, M., Shanahan, J., \& Signorielli, N. (2009). Growing up with television: Cultivation processes. In J. Bryant \& M. B. Oliver (Eds.), Media effects: Advances in theory and research (pp. 34-49). New York: Routledge.

Mulgrew, K. E. (2016). Fitspiration' photos cause body image strain. Retrieved from http://www.usc.edu.au/explore/usc-news-exchange/news-archive/2016/january/Fitspiration-photoscause-body-image-strain

Mulgrew, K. E., \& Hennes, S. M. (2014). The effect of functionality- and aesthetic-focused images on Australian women's body satisfaction. Sex Roles. doi:10.1007/s11199-014-0440-2

National Eating Disorders Association. (2016). Responsible media coverage of eating disorders. Retrieved from https://www.nationaleatingdisorders.org/responsible-media-coverage-eating-disorders

National Eating Disorders Association \& California State University. (n. d.). Get Real: Digital Media Literacy Toolkit.

Retrieved from http://www.nationaleatingdisorders.org/sites/default/files/Toolkits/GetRealToolkit.pdf

Perloff, R. M. (2014). Social media effects on young women's body image concerns: Theoretical perspectives and an agenda for research. Sex Roles. doi:10.1007/s11199-014-0384-6

Pinterest Acceptable Use Policy. (2016). Retrieved from https://about.pinterest.com/en/acceptable-usepolicy

Prieler, M., \& Choi, J. (2014). Broadening the scope of social media effect research on body image concerns. Sex Roles, 71, 378-388.

Smolak, L., \& Thompson, J. K. (2009). Body image, eating disorders, and obesity in children and adolescents. In L. Smolak \& J. K. Thompson (Eds.), Body image, eating disorders, and obesity in youth: Assessment, prevention, and treatment (pp. 3-14). Washington, DC: APA.

Snapchat Community Guidelines. (2016). Retrieved from https://support.snapchat.com/enUS/a/guidelines

Spiegel, A. R. (2013). Here's How Social Media "Thinspiration" Bans Are Actually Working Out. Retrieved from https://www.buzzfeed.com/verymuchso/heres-how-social-media-thinspiration-bans-area?utm_term=.cbEMKM9xN\#.fwQeze1BA 
Statista. (2016a). Reach of leading social media and networking sites used by teenagers and young adults in the United States as of February 2016. Retrieved from http://www.statista.com/statistics/199242/socialmedia-and-networking-sites-used-by-us-teenagers/

Statista. (2016b). Leading social networks worldwide as of April 2016, active users (in millions). Retrieved from http://www.statista.com/statistics/272014/global-social-networks-ranked-by-number-of-users/

Stieben, D. (2013). Why Am I Seeing This Ad? How Social Media Ads Target You. Retrieved from http://www.makeuseof.com/tag/why-am-i-seeing-this-ad-how-social-media-ads-target-you/

Strelan P., Mehaffey, S. J., \& Tiggemann, M. (2003). Self-objectification and esteem in young women: The mediating role of reasons for exercise. Sex Roles, 48, 89-95.

The Pro Ana Lifestyle Forever. (n. d.). Retrieved from https://theproanalifestyleforever.wordpress.com/religionandlifestyle/

Thompson, J. K., Heinberg, L. J., Altabe, M., \& Tantleff-Dunn, S. (1999). Exacting beauty: Theory, assessment, and treatment of body image disturbance. Washington, DC: APA.

Tiggemann, M. (2011). Sociocultural perspectives on human appearance and body image. In T. F. Cash \& L. Smolak (Eds.), Body image: A handbook of science, practice, and prevention (pp. 12-19). New York: Guilford Press.

Tiggemann, M., \& Zaccardo, M. (2015). Exercise to be fit, not skinny: The effect of Fitspiration imagery on women's body image. Body Image, 15, 61-67. Retrieved from http://www.

sciencedirect.com/science/article/pii/S1740144515000893

Tiggemann, M., \& Zaccardo, M. (2016). Strong is the new skinny: A content analysis of \#Fitspiration images on Instagram. Journal of Health Psychology,1-9. doi:10.1177/1359105316639436

Tufekci, Z. (2008). Grooming, gossip, Facebook and MySpace. Information, Communication, and Society, 11(4), 544-564.

Tumblr Community Guidelines. (2015). Retrieved from https://www.tumblr.com/policy/en/community

The Twitter Rules. (2016). Retrieved from https://support.twitter.com/articles/18311

U.S. Department of Health and Human Services. (2010). National action plan to improve health

literacy. Retrieved from

http://www.health.gov/communication/hlactionplan/pdf/Health_Literacy_Action_Plan.pdf

Vaterlaus, M., Patten, E. V., Rochec, C., \& Young, J. A. (2015). \#Gettinghealthy: The perceived influence of social media on young adult health behaviors. Computers in Human Behavior, 45, 151-157.

Vogel, A. (2015). Positive Body Image Posting Guide. Retrieved from http://www.idealfit.com/fitnessLibrary/positive-body-image-posting-guide

Williams, R. J., \& Ricciardelli, L. A. (2014). Social media and body image concerns: Further considerations and broader perspectives. Sex Roles, 71, 389-392. doi:10.1007/s11199-014-0429-x

Zalta, A. K., \& Keel, P. K. (2006). Peer influence on bulimic symptoms in college students. Journal of Abnormal Psychology, 115(1), 185-189. Retrieved from http://dx.doi.org/10.1037/0021-843X.115.1.185 
Zhao, S., Grasmuck, S., \& Martin, J. (2008). Identity construction on Facebook: Digital empowerment in anchored relationships. Computers in Human Behavior, 24, 1816-1836. doi:10.1016/j.chb.2008.02.012

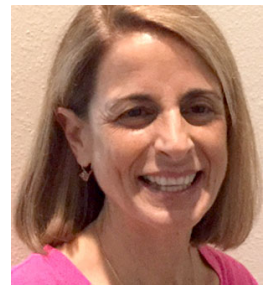

Joanne M. Crossman is a Professor of Education in the School of Education and Social Services at Saint Leo University in Florida. She earned an Ed.D. in Educational Leadership, C.A.G.S. in Higher Education Curriculum/Administration, M.Ed. in Health Education, and B.A. in Communication, and numerous education certifications. Dr. Crossman has delivered more than 60 conference presentations. Her research interests include pedagogy, assessment, and wellness education. During her 29 years as an educator, she has provided oversight of curriculum and instruction, assessment, professional development, chaired critical committees, and mentored beginning teachers. She received a number awards as an educator, Toastmaster, and for community service work, championing student outreach initiatives with more than 50 not-for-profit organizations. 